\title{
Variation in Floral Induction Requirements of Hibiscus sp.
}

\author{
Ryan M. Warner ${ }^{1}$ and John E. Erwin ${ }^{2}$ \\ Department of Horticultural Science, University of Minnesota, 1970 Folwell Ave., Saint Paul, MN 55108-6007
}

\begin{abstract}
AdDITIONAL INDEX words. Malvaceae, flower initiation, flower diameter, branching, irradiance, photoperiod, temperature
Abstract. Thirty-six Hibiscus L. species were grown for 20 weeks under three lighting treatments at 15,20, or $25 \pm 1.5{ }^{\circ} \mathrm{C}$ air temperature to identify flowering requirements for each species. In addition, species were subjectively evaluated to identify those species with potential ornamental significance based on flower characteristics and plant form. Lighting treatments were 9 hour ambient light (St. Paul, Minn., November to May, $45^{\circ} \mathrm{N}$ ), ambient light plus a night interruption using incandescent lamps $\left(2 \mu \mathrm{mol} \cdot \mathrm{m}^{-2} \cdot \mathrm{s}^{-1} ; 2200\right.$ to $\left.0200 \mathrm{HR}\right)$, or ambient light plus 24 -hour supplemental lighting from high-pressure sodium lamps $\left(100 \mu \mathrm{mol} \cdot \mathrm{m}^{-2} \cdot \mathrm{s}^{-1}\right)$. Five day-neutral, six obligate short-day, six facultative short-day, three obligate long-day, and one facultative long-day species were identified. Fifteen species did not flower. Temperature and lighting treatments interacted to affect leaf number below the first flower and/or flower diameter on some species. Hibiscus acetosella Welw. ex Hiern, H. cisplatinus St.-Hil., H. radiatus Cav., and $\boldsymbol{H}$. trionum $\mathbf{L}$. were selected as potential new commercially significant ornamental species.
\end{abstract}

Hibiscus is the largest genus in the Malvaceae family (250 to 300 species; Bates, 1965). Hibiscus sp. are primarily tropical in origin, although several species are native to temperate and warm temperate regions (Table 1). Although the genus is quite large, only $H$. rosa-sinensis L. (Chinesehibiscus), H. syriacus L.(Rose-of-Sharon), H. moscheutos L., and hybrids developed by crossing $H$. coccineus (Medic.) Walt., H. laevis All., and H. moscheutos are grown commercially as ornamental plants.

Cultivated Hibiscus are known for their large flowers that range in color from yellow to white and shades of pink and red. Flower diameter of some herbaceous perennial cultivars can be up to $30 \mathrm{~cm}$ (R. Warner, personal observation).

Commercially grown Hibiscus are both day-neutral (H. rosasinensis) (Dole and Wilkins, 1999) and long-day plants [H. syriacus (Thomas and Vince-Prue, 1997), H. moscheutos (Runkle et al., 1996)]. Hibiscus coccineus flowers naturally in July (Bates, 1965), suggesting that it may also be a long-day plant. Hibiscus hiernianus Exell \& Mend., H. mastersianus Hiern., and H. mechowii Garcke are short-day plants (Menzel et al., 1986). However, little information is available on the floral induction requirements of Hibiscus species not commercially cultivated.

The popularity of Hibiscus presently being cultivated suggests other Hibiscus sp. may have commercial potential as ornamental plants. Therefore, the objectives of this research were to 1) determine environmental requirements for flowering of Hibiscus sp. and 2) identify potentially significant new ornamental species. Environmental treatments were selected to cover a wide range of thermoand photoperiodic possibilities to maximize the number of species that would be induced to flower.

\section{Materials and Methods}

Seeds of 36 Hibiscus sp. (Table 1 ) were soaked for $12 \mathrm{~h}$ in $\approx 75$ ${ }^{\circ} \mathrm{C}$ water that was allowed to cool to room temperature $\left(\approx 22^{\circ} \mathrm{C}\right)$ (Nau, 1996) on 23 Nov. 1996. The experiment was repeated on 24

Received for publication 25 Feb. 2000. Accepted for publication 28 Dec. 2000. Minnesota Agricultural Experiment Station scientific journal series paper 981210042. The authors wish to acknowledge the Minnesota Commercial Flower Growers Association, Minnesota Agricultural Experiment Station, Minnesota Extension Service, Widmer Fund, and Ohio Floriculture Foundation for financial support of this project. The cost of publishing this paper was defrayed in part by the payment of page charges. Under postal regulations, this paper therefore must be hereby marked advertisement solely to indicate this fact.

${ }^{1}$ Graduate research assistant.

${ }^{2}$ Associate professor.
Oct. 1997. Seeds that did not imbibe after soaking were scarified manually. Seeds were then sown in $25-\mathrm{mL}$ cells in a soilless media (Germination Mix, Strong-Lite Horticultural Products, Pine Bluff, Ark.) and covered with $\approx 2$ to $4 \mathrm{~mm}$ of vermiculite (Premium Grade Medium Vermiculite, Strong-Lite Horticultural Products). Planting depth of each species varied and was $\approx 1.5$ times the minimum diameter of a seed. Seeds were germinated under intermittent mist ( $6 \mathrm{~s}$ of mist every $10 \mathrm{~min}$, from 0700 to $1800 \mathrm{HR}$ daily) at $23 \pm 1{ }^{\circ} \mathrm{C}$ air temperature. Seedlings were removed from the mist and transplanted into $450-\mathrm{mL}$ pots in a soilless media (Universal Mix, Strong-Lite Horticultural Products) when cotyledons were parallel to the media surface $(\approx 2$ to $3 \mathrm{~d}$ after emergence from the media). Transplanted seedlings were then placed in a greenhouse maintained at $20 \pm 1^{\circ} \mathrm{C}$ air temperature under ambient daylight conditions (December 1996; November 1997; St. Paul, Minn.) for $7 \mathrm{~d}$, after which seedlings were moved and environmental treatments were initiated.

Environmental treatments consisted of combinations of three air temperature treatments $\left(15,20\right.$, or $\left.25 \pm 1.5^{\circ} \mathrm{C}\right)$, and three lighting treatments. Lighting treatments within each temperature were: short-day (SD; $9 \mathrm{~h}$ of ambient light, plants covered with opaque black cloth daily from 1700 to $0800 \mathrm{HR}$; temperature under the black cloth was not recorded), night-interruption [NI; ambient daylight plus $2 \mu \mathrm{mol} \cdot \mathrm{m}^{-2} \cdot \mathrm{s}^{-1}$ irradiance from 2200 to $0200 \mathrm{HR}$, using incandescent lamps (Sylvania Directlite 100W, GTE Products Corp., Salem, Mass.)], or continuous lighting [CT; ambient daylight plus continuous $100 \mu \mathrm{mol} \cdot \mathrm{m}^{-2} \cdot \mathrm{s}^{-1}$ supplemental lighting from high-pressure sodium lamps (Lucolux LU400, GeneralElectric, Cleveland, Ohio)]. Plants were fertilized weekly with $14.3 \mathrm{~mm} \mathrm{~N}, 0 \mathrm{~mm} P$, and $6.5 \mathrm{~mm}$ $\mathrm{K}$, supplied by $\mathrm{Ca}\left(\mathrm{NO}_{3}\right)_{2}$ and $\mathrm{KNO}_{3}$. Every fourth week, plants were fertilized with $14.3 \mathrm{~mm} \mathrm{~N}, 0.72 \mathrm{~mm}$ P, $6.5 \mathrm{~mm} \mathrm{~K}, 1.67 \mathrm{~mm} \mathrm{Ca}, 1.1$ mM Mg, plus trace amounts of micronutrients (Peter's Excel 15N2.2P-12.5K Cal-Mg, The Scott's Co., Marysville, Ohio).

Data were collected at anthesis or after 20 weeks on leaf number below the first flower, plant height (from the media surface to the apical meristem), height to the first flowering node, first flower diameter, lateral shoot number (lateral shoots $\geq 2.5 \mathrm{~cm}$ ), and visible flower bud number ( $\geq 1 \mathrm{~cm}$ in length). Plants with visible flower buds after 20 weeks remained in environmental treatments until anthesis. Potential commercial ornamental significance was evaluated subjectively based on floral characteristics, including flower size, flower color, flower number, whether plants flowered continuously, leaf and stem characteristics, and overall plant architecture.

The experiment was replicated twice over time, with each 
treatment consisting of four plants per replicate. Plants were completely randomized within each lighting/temperature treatment combination. Means were separated using Tukey's HSD test $(P<0.05)$ using the SPSS statistical analysis program (SPSS, Inc., 1997).

\section{Results}

FLOWER INDUCTION AND DEVELOPMENT. Lighting and temperature treatments interacted with species to affect leaf number below the first flower. For some species there were insufficient plants for all treatments, so plants were placed in the 20 and $25^{\circ} \mathrm{C}$ temperature treatments, or under SD and NI in the $20^{\circ} \mathrm{C}$ temperature treatment only (Tables 2 and 3 ). Fifteen species did not flower under any environmental treatment (Table 1). All species that flowered had a single flower in each leaf axil. Six species, $H$. acetosella, $H$. cannabinus, $H$. engleri, $H$. mastersianus, $H$. nigricaulis, and $H$. schinzii flowered with a lower leaf number under the SD lighting treatment than under the NI lighting treatment (Table 2).

Hibiscus asper, H. meeusei, H. physaloides, H. sabdariffa, and $H$. surattensis flowered only under the SD lighting treatment (Tables 2 and 3). Hibiscus radiatus flowered under the SD lighting treatment at all three temperatures, but flowered under NI lighting only when grown at $15^{\circ} \mathrm{C}$ (Tables 2 and 3). Hibiscus surattensis flowered with a similar leaf number below the first flower when grown under the SD or CT lighting treatments (Table 2), but did not flower when grown under the NI lighting treatment.

Species that flowered at $20^{\circ} \mathrm{C}$ regardless of lighting treatment were $H$. calyphyllus, $H$. cisplatinus, $H$. costatus, $H$. lunarriifolius, and $H$. meraukensis (Tables 2 and 3 ). These species flowered with a similar leaf number below the first flower under either the SD or NI lighting treatments.

Hibiscus aculeatus, $H$. laevis, and $H$. moscheutos required a photoperiod inductive for flowering to grow, i.e., for continued

Table 1. Hibiscus sp., source, native range, and native range latitude of species evaluated in this experiment.

\begin{tabular}{|c|c|c|c|}
\hline Species & Source & $\begin{array}{l}\text { Native } \\
\text { range }\end{array}$ & $\begin{array}{l}\text { Approximate } \\
\text { latitude }\left(^{\circ}\right)\end{array}$ \\
\hline H. acetosella Welw. ex. Hiern & $\mathrm{PI}^{\mathrm{z}} 260997$ & Angola & $6-17 \mathrm{~S}$ \\
\hline H. aculeatus Walt. & Commercial & Florida to Louisiana and North Carolina & $30-35 \mathrm{~N}$ \\
\hline H. arnhemensis F.D. Wilson ${ }^{\mathrm{y}}$ & PI 585151 & N. Territory, Australia(Wilson, 1974) & $12-25 \mathrm{~S}$ \\
\hline H. arnottianus A. Gray ${ }^{\mathrm{y}}$ & Commercial & Hawaii, endemic to Oahu & $23 \mathrm{~N}$ \\
\hline H. asper Hook f. & PI 585165 & Collected in Uganda & $4 \mathrm{~N}-2 \mathrm{~S}$ \\
\hline H. biseptus ${ }^{\mathrm{y}}$ & Commercial & S. Arizona and New Mexico (Rickett, 1970) & $30-33 \mathrm{~N}$ \\
\hline H. calyphyllus Cav. & PI 364895 & Tropical and South Africa & $0-30 \mathrm{~S}$ \\
\hline H. cannabinus $\mathrm{L}$. & Commercial & Probably Tropical Africa & $23 \mathrm{~N}-23 \mathrm{~S}$ \\
\hline H. cisplatinus St.-Hil. ${ }^{\mathrm{x}}$ & PI 372212 & Argentina, Paraguay, Uruguay, and S. Brazil & $23-33 \mathrm{~S}$ \\
\hline H. coccineus Walt. ${ }^{\mathrm{y}}$ & Commercial & Georgia and S. Florida & $28-33 \mathrm{~N}$ \\
\hline H. costatus A. Rich ${ }^{\mathrm{x}}$ & PI 265239 & Cuba, Mexico, C. America (Menzel et al., 1983) & $12-23 \mathrm{~N}$ \\
\hline H. denudatus Benth. ${ }^{\mathrm{y}}$ & Commercial & California to Texas and Northern Mexico & $30-35 \mathrm{~N}$ \\
\hline H. diversifolius Jacq. ${ }^{\mathrm{y}}$ & PI 263965 & Africa and Asian Tropics & $23 \mathrm{~N}-23 \mathrm{~S}$ \\
\hline H. dongolensis Caill. ex Del. ${ }^{\mathrm{xy}}$ & PI 364899 & Collected in South Africa & $23-32 \mathrm{~S}$ \\
\hline H. engleri Schumann ${ }^{\mathrm{x}}$ & PI 364901 & Collected in South Africa & $23-32 \mathrm{~S}$ \\
\hline H. fallax Craven \& F.D. Wilson ${ }^{y}$ & PI 595768 & Collected in N. Territory Australia & $12-25 \mathrm{~S}$ \\
\hline H. furcellatus Desr. ${ }^{\mathrm{y}}$ & PI 585122 & West Indies, Central, and South America, Hawaii & $0-25 \mathrm{~N}$ \\
\hline H. greenwayi Baker f. ${ }^{\mathrm{y}}$ & PI 405056 & Collected in Kenya & $4 \mathrm{~S}-4 \mathrm{~N}$ \\
\hline H. laevis All. & Commercial & Eastern United States & $27-42 \mathrm{~N}$ \\
\hline H. lunariifolius Willd. ${ }^{\mathrm{x}}$ & PI 369345 & Collected in Uganda & $2 \mathrm{~S}-4 \mathrm{~N}$ \\
\hline H. mastersianus Hiern ${ }^{\mathrm{x}}$ & PI 585154 & Collected in Zambia & $8-18 \mathrm{~S}$ \\
\hline H. meeusei Exell & PI 344232 & Collected in Zambia & $8-18 \mathrm{~S}$ \\
\hline H. meraukensis Hochr. & PI 585121 & Western Australia (Wilson, 1974) & $15-35 \mathrm{~S}$ \\
\hline H. moscheutos L. & Commercial & Eastern United States & $27-42 \mathrm{~N}$ \\
\hline H. mutabilis L. ${ }^{\mathrm{y}}$ & Commercial & Probably S. China and Taiwan & $20-30 \mathrm{~N}$ \\
\hline H. nigricaulis Baker $\mathrm{f}^{\mathrm{x}}$ & PI 585155 & Collected in Zambia & $8-18 \mathrm{~S}$ \\
\hline H. physaloides Guill. & PI 364904 & Collected in South Africa & $23-32 \mathrm{~S}$ \\
\hline H. radiatus Cav. & PI 585159 & Asian Tropics & $0-23 \mathrm{~N}$ \\
\hline H. rostellatus Guill. \& Perr. ${ }^{y}$ & PI 344219 & Central Africa (Menzel and Wilson, 1963) & $10 \mathrm{~S}-20 \mathrm{~N}$ \\
\hline H. sabdariffa $\mathrm{L}$. & PI 500741 & Probably Tropical Africa & $23 \mathrm{~S}-23 \mathrm{~N}$ \\
\hline H. schinzii Gurke. ${ }^{\mathrm{x}}$ & PI 500714 & Southern Africa & $10-30 \mathrm{~S}$ \\
\hline H. surattensis $\mathrm{L}$. & PI 585138 & Central Africa (Menzel and Wilson, 1963) & $10 \mathrm{~S}-20 \mathrm{~N}$ \\
\hline H. taiwaniana ${ }^{y}$ & Commercial & China & $20-40 \mathrm{~N}$ \\
\hline H. tiliaceus L. ${ }^{\mathrm{y}}$ & Commercial & Old and New World Tropics & $23 \mathrm{~S}-23 \mathrm{~N}$ \\
\hline H. trionum L. Sunnyday & Commercial & Widespread in Europe, Africa, Asia, and Australia & \\
\hline H. zonatus F. Muell. ${ }^{\mathrm{y}}$ & PI 585136 & N. Territory, Australia (Wilson, 1974) & $12-25 \mathrm{~S}$ \\
\hline
\end{tabular}

${ }^{\mathrm{z} U S D A}$ plant introduction (PI) number.

${ }^{y}$ Species did not flower under any environmental treatment in this experiment.

${ }^{x}$ Seeds were scarified manually. 
Table 2. Effect of temperature and lighting treatment on leaf number below the first flower of Hibiscus sp. Species are grouped into the appropriate photoperiodic response group. Numerals in parentheses represent leaf number at the end of the experiment on plants that did not flower. Plants were grown under $9 \mathrm{~h}$ ambient light (St. Paul, Minn., from November to May) (SD), ambient light plus a $2 \mu \mathrm{mol} \cdot \mathrm{m}^{-2} \cdot \mathrm{s}^{-1} \mathrm{night}$ interruption using incandescent lamps from 2200 to $0200 \mathrm{HR}(\mathrm{NI})$, or ambient light plus $100 \mu \mathrm{mol} \cdot \mathrm{m}^{-2} \cdot \mathrm{s}^{-1}$ continual supplemental lighting from high pressure sodium lamps (CT).

\begin{tabular}{|c|c|c|c|c|}
\hline \multirow[b]{2}{*}{ Species } & \multirow{2}{*}{$\begin{array}{c}\text { Air } \\
\text { temp }\left({ }^{\circ} \mathrm{C}\right)\end{array}$} & \multicolumn{3}{|c|}{ Leaf no. } \\
\hline & & SD & NI & CT \\
\hline & & \multicolumn{3}{|c|}{ Facultative short-day } \\
\hline \multirow[t]{2}{*}{ H. acetosella } & 20 & $17^{z} a^{y} A^{x}$ & $38 \mathrm{bA}$ & $37 \mathrm{~b}$ \\
\hline & 25 & $15 \mathrm{aA}$ & $47 \mathrm{bB}$ & $\mathrm{na}^{\mathrm{w}}$ \\
\hline \multirow[t]{2}{*}{ H. cannabinus ${ }^{\mathrm{v}}$} & 15 & $17 \mathrm{aA}$ & $26 \mathrm{bA}$ & $27 \mathrm{bA}$ \\
\hline & 20 & $25 \mathrm{aB}$ & $34 \mathrm{bB}$ & $38 \mathrm{bB}$ \\
\hline H. engleri & 20 & $10 \mathrm{a}$ & $19 \mathrm{~b}$ & na \\
\hline \multirow[t]{2}{*}{ H. mastersianus } & 20 & $7 \mathrm{aA}$ & $10 \mathrm{bA}$ & $14 \mathrm{c}$ \\
\hline & 25 & $12 \mathrm{aB}$ & $23 \mathrm{bB}$ & $(38)$ \\
\hline \multirow[t]{2}{*}{ H. nigricaulis } & 20 & $6 \mathrm{aA}$ & $12 \mathrm{bA}$ & $17 \mathrm{cA}$ \\
\hline & 25 & $10 \mathrm{aB}$ & $22 \mathrm{bB}$ & $24 \mathrm{bB}$ \\
\hline \multirow[t]{2}{*}{ H. schinzii } & 20 & $8 \mathrm{a}$ & $13 \mathrm{~b}$ & na \\
\hline & & \multicolumn{3}{|c|}{ Obligate short-day } \\
\hline \multirow{3}{*}{ H. asper } & 15 & $7 \mathrm{~A}$ & (18) & $(21)$ \\
\hline & 20 & $7 \mathrm{~A}$ & (20) & (20) \\
\hline & 25 & $23 \mathrm{~B}$ & (25) & (26) \\
\hline \multirow[t]{2}{*}{ H. meeusei } & 20 & $6 \mathrm{~A}$ & (17) & na \\
\hline & 25 & $7 \mathrm{~A}$ & (21) & na \\
\hline \multirow[t]{2}{*}{ H. physaloides } & 20 & $15 \mathrm{~A}$ & (22) & (24) \\
\hline & 25 & $24 \mathrm{aB}$ & (27) & (29) \\
\hline \multirow[t]{3}{*}{$H$. radiatus $^{\mathrm{u}}$} & 15 & $11 \mathrm{aA}$ & $21 \mathrm{~b}$ & $24 \mathrm{bA}$ \\
\hline & 20 & $16 \mathrm{aA}$ & (31) & $32 \mathrm{bB}$ \\
\hline & 25 & $11 \mathrm{aA}$ & (41) & $(45)$ \\
\hline \multirow[t]{3}{*}{ H. sabdariffa } & 15 & Dead & Dead & Dead \\
\hline & 20 & $8 \mathrm{~A}$ & (16) & $(16)$ \\
\hline & 25 & $7 \mathrm{~A}$ & (21) & (19) \\
\hline \multirow[t]{4}{*}{ H. surattensis } & 15 & Dead & Dead & (9) \\
\hline & 20 & $10 \mathrm{aA}$ & $(20)$ & $10 \mathrm{aA}$ \\
\hline & 25 & $13 \mathrm{aB}$ & (31) & $13 \mathrm{aB}$ \\
\hline & & \multicolumn{3}{|c|}{ Day neutral } \\
\hline H. calyphyllus & 20 & $15 \mathrm{a}$ & $13 \mathrm{a}$ & $15 \mathrm{a}$ \\
\hline H. cisplatinus & 20 & $21 \mathrm{a}$ & $21 \mathrm{a}$ & $21 \mathrm{a}$ \\
\hline H. costatus & 20 & $9 \mathrm{a}$ & $9 a$ & na \\
\hline H. lunariifolius & 20 & $13 \mathrm{a}$ & $13 \mathrm{a}$ & na \\
\hline \multirow[t]{2}{*}{ H. meraukensis } & 20 & $9 \mathrm{a}$ & $9 \mathrm{a}$ & $8 \mathrm{a}$ \\
\hline & & \multicolumn{3}{|c|}{ Facultative long-day } \\
\hline \multirow[t]{4}{*}{ H. trionum } & 15 & $7 \mathrm{aA}$ & $8 \mathrm{aA}$ & $7 \mathrm{aA}$ \\
\hline & 20 & $16 \mathrm{cB}$ & $10 \mathrm{bB}$ & $6 \mathrm{aA}$ \\
\hline & 25 & $22 \mathrm{cC}$ & $11 \mathrm{bB}$ & $6 \mathrm{aA}$ \\
\hline & & & gate lons & \\
\hline \multirow[t]{2}{*}{ H. aculeatus } & 20 & Dead & $28 \mathrm{aB}$ & $33 \mathrm{aB}$ \\
\hline & 25 & Dead & $22 \mathrm{aA}$ & $25 \mathrm{aA}$ \\
\hline \multirow[t]{2}{*}{ H. laevis } & 20 & $g c^{t}$ & $24 \mathrm{aA}$ & $24 \mathrm{aA}$ \\
\hline & 25 & $\mathrm{gc}$ & $25 \mathrm{aA}$ & $24 \mathrm{aA}$ \\
\hline \multirow[t]{3}{*}{ H. moscheutos } & 15 & $\mathrm{gc}$ & $23 \mathrm{~A}$ & na \\
\hline & 20 & $\mathrm{gc}$ & $24 \mathrm{aA}$ & $25 \mathrm{aA}$ \\
\hline & 25 & $\mathrm{gc}$ & $23 \mathrm{aA}$ & $24 \mathrm{aA}$ \\
\hline
\end{tabular}

${ }^{\mathrm{z}}$ Numerals represent treatment means.

'Lower case letters indicate mean separation within a species and temperature, across lighting treatment, by Tukey's studentized range test, $P<0.05$.

xUpper case letters indicate mean separation within a species and lighting treatment, across temperature, by Tukey's studentized range test, $P<0.05$.

wPlants were not grown in this treatment.

velants became too tall to maintain in $25^{\circ} \mathrm{C}$ treatments.

uFacultative short-day plants at $15^{\circ} \mathrm{C}$.

${ }^{\mathrm{t}}$ Growth ceased after plants unfolded $\approx 10$ leaves. 
Table 3. Effect of temperature and lighting treatment on days to first open flower of Hibiscus sp. Species are grouped into the appropriate photoperiodic response group. Plants were grown under $9 \mathrm{~h}$ ambient light (St. Paul, Minn., from November to May) (SD), ambient light plus a $2 \mu \mathrm{mol} \cdot \mathrm{m}^{-2} \cdot \mathrm{s}^{-1}$ night interruption using incandescent lamps from 2200 to $0200 \mathrm{HR}(\mathrm{NI})$, or ambient light plus $100 \mu \mathrm{mol} \cdot \mathrm{m}^{-2} \cdot \mathrm{s}^{-1} \mathrm{continual}$ supplemental lighting from high pressure sodium lamps (CT).

\begin{tabular}{|c|c|c|c|c|}
\hline \multirow[b]{2}{*}{ Species } & \multirow{2}{*}{$\begin{array}{c}\text { Air } \\
\text { temp }\left({ }^{\circ} \mathrm{C}\right)\end{array}$} & \multicolumn{3}{|c|}{ Days to first open flowr } \\
\hline & & SD & $\mathrm{NI}$ & $\mathrm{CT}$ \\
\hline & & \multicolumn{3}{|c|}{ Facultative short-day } \\
\hline \multirow[t]{2}{*}{ H. acetosella } & 20 & $103^{z} a^{y} B^{x}$ & $159 \mathrm{bA}$ & $155 \mathrm{~b}$ \\
\hline & 25 & $69 \mathrm{aA}$ & $152 \mathrm{bA}$ & $\mathrm{na}^{\mathrm{w}}$ \\
\hline \multirow[t]{2}{*}{ H. cannabinus ${ }^{\mathrm{v}}$} & 15 & $166 \mathrm{aB}$ & $195 \mathrm{bB}$ & $192 \mathrm{bB}$ \\
\hline & 20 & $145 \mathrm{aA}$ & $177 \mathrm{bA}$ & $169 \mathrm{bA}$ \\
\hline H. engleri & 20 & $57 \mathrm{a}$ & $85 \mathrm{~b}$ & na \\
\hline \multirow[t]{2}{*}{ H. mastersianus } & 20 & $40 \mathrm{aA}$ & $49 \mathrm{bA}$ & $52 \mathrm{~b}$ \\
\hline & 25 & $41 \mathrm{aA}$ & $63 \mathrm{bB}$ & na \\
\hline \multirow[t]{2}{*}{ H. nigricaulis } & 20 & $46 \mathrm{aA}$ & $67 \mathrm{bA}$ & $75 \mathrm{bA}$ \\
\hline & 25 & $45 \mathrm{aA}$ & $92 \mathrm{bB}$ & $94 \mathrm{bB}$ \\
\hline \multirow[t]{2}{*}{ H. schinzii } & 20 & $60 \mathrm{a}$ & $89 \mathrm{~b}$ & na \\
\hline & & & gate shor & \\
\hline \multirow[t]{3}{*}{ H. asper } & 15 & $66 \mathrm{~B}$ & $\operatorname{dnf}^{u}$ & $\operatorname{dnf}$ \\
\hline & 20 & $52 \mathrm{~A}$ & $\operatorname{dnf}$ & $\operatorname{dnf}$ \\
\hline & 25 & $109 \mathrm{C}$ & $\operatorname{dnf}$ & $\operatorname{dnf}$ \\
\hline \multirow[t]{2}{*}{ H. meeusei } & 20 & $54 \mathrm{~B}$ & $\operatorname{dnf}$ & na \\
\hline & 25 & $43 \mathrm{~A}$ & $\operatorname{dnf}$ & na \\
\hline \multirow[t]{2}{*}{ H. physaloides } & 20 & $59 \mathrm{~A}$ & $\operatorname{dnf}$ & $\operatorname{dnf}$ \\
\hline & 25 & $70 \mathrm{~B}$ & $\operatorname{dnf}$ & $\operatorname{dnf}$ \\
\hline \multirow[t]{3}{*}{ H. radiatus ${ }^{\mathrm{t}}$} & 15 & $119 \mathrm{aC}$ & $175 \mathrm{~b}$ & $177 \mathrm{bB}$ \\
\hline & 20 & $88 \mathrm{aA}$ & $\operatorname{dnf}$ & $122 \mathrm{aA}$ \\
\hline & 25 & $60 \mathrm{~A}$ & $\operatorname{dnf}$ & $\operatorname{dnf}$ \\
\hline \multirow[t]{2}{*}{ H. sabdariffa } & 20 & $74 \mathrm{~B}$ & $\operatorname{dnf}$ & $\operatorname{dnf}$ \\
\hline & 25 & $56 \mathrm{~A}$ & $\operatorname{dnf}$ & $\operatorname{dnf}$ \\
\hline \multirow[t]{3}{*}{ H. surattensis } & 20 & $71 \mathrm{aB}$ & $\operatorname{dnf}$ & $68 \mathrm{aA}$ \\
\hline & 25 & $53 \mathrm{aA}$ & $\operatorname{dnf}$ & $47 \mathrm{aA}$ \\
\hline & & \multicolumn{3}{|c|}{ Day neutral } \\
\hline H. calyphyllus & 20 & $155 \mathrm{a}$ & $167 \mathrm{a}$ & $160 \mathrm{a}$ \\
\hline H. cisplatinus & 20 & $144 \mathrm{a}$ & $147 \mathrm{a}$ & $141 \mathrm{a}$ \\
\hline H. costatus & 20 & $82 \mathrm{a}$ & $86 a$ & na \\
\hline H. lunariifolius & 20 & $61 \mathrm{a}$ & $66 \mathrm{a}$ & na \\
\hline \multirow[t]{2}{*}{ H. meraukensis } & 20 & $97 \mathrm{a}$ & $101 \mathrm{a}$ & $98 \mathrm{a}$ \\
\hline & & \multicolumn{3}{|c|}{ Facultative long-day } \\
\hline \multirow[t]{4}{*}{ H. trionum } & 15 & $110 \mathrm{cA}$ & $92 \mathrm{bC}$ & $61 \mathrm{aC}$ \\
\hline & 20 & $109 \mathrm{cA}$ & $79 \mathrm{bB}$ & $43 \mathrm{aB}$ \\
\hline & 25 & $107 \mathrm{cA}$ & $68 \mathrm{bA}$ & $36 \mathrm{aA}$ \\
\hline & & & igate long & \\
\hline \multirow[t]{2}{*}{ H. aculeatus } & 20 & Dead & $160 \mathrm{aB}$ & $151 \mathrm{aB}$ \\
\hline & 25 & Dead & $131 \mathrm{bA}$ & $99 \mathrm{aA}$ \\
\hline \multirow[t]{2}{*}{ H. laevis } & 20 & $\mathrm{gc}^{\mathrm{s}}$ & $135 \mathrm{aB}$ & $131 \mathrm{aB}$ \\
\hline & 25 & $\mathrm{gc}$ & $119 \mathrm{aA}$ & $115 \mathrm{aA}$ \\
\hline \multirow[t]{3}{*}{ H. moscheutos } & 15 & $\mathrm{gc}$ & $133 \mathrm{C}$ & na \\
\hline & 20 & $\mathrm{gc}$ & $112 \mathrm{aB}$ & $105 \mathrm{aB}$ \\
\hline & 25 & $\mathrm{gc}$ & $99 \mathrm{aA}$ & $90 \mathrm{aA}$ \\
\hline
\end{tabular}

${ }_{\mathrm{z}}$ Numerals represent treatment means.

'Lower case letters indicate mean separation within a species and temperature, across lighting treatment, by Tukey's studentized range test, $P<0.05$.

xUpper case letters indicate mean separation within a species and lighting treatment, across temperature, by Tukey's studentized range test, $P<0.05$.

wPlants were not grown in this treatment.

vPlants became too tall to maintain in $25^{\circ} \mathrm{C}$ treatments.

uplants did not flower in this treatment.

tFacultative short-day plants at $15^{\circ} \mathrm{C}$.

${ }^{\mathrm{s}}$ Growth ceased after plants unfolded $\approx 10$ leaves. 
Table 4. Comparison of flower color and diameter of 21 Hibiscus sp. grown under the lighting treatment that resulted in earliest flowering at $20^{\circ} \mathrm{C}$.

\begin{tabular}{llc}
\hline \hline Species & $\begin{array}{l}\text { Flower color } \\
\text { (center color) }\end{array}$ & $\begin{array}{c}\text { Flower diam } \\
(\mathrm{mm})\end{array}$ \\
\hline H. acetosella & Yellow (maroon) & $83^{\mathrm{z}} \mathrm{h}^{\mathrm{y}}$ \\
H. aculeatus & Yellow (maroon) & $75 \mathrm{~g}$ \\
H. asper & Yellow (maroon) & $52 \mathrm{e}$ \\
H. calyphyllus & Yellow (red-brown) & $47 \mathrm{~d}$ \\
H. cannabinus & Cream (maroon) & $101 \mathrm{i}$ \\
H. cisplatinus & Pink & $107 \mathrm{j}$ \\
H. costatus & Pink & $51 \mathrm{e}$ \\
H. engleri & Yellow (brown) & $33 \mathrm{bc}$ \\
H. laevis & Pink (maroon) & $82 \mathrm{~h}$ \\
H. lunariifolius & Yellow (maroon) & $64 \mathrm{f}$ \\
H. mastersianus & Yellow (maroon) & $55 \mathrm{e}$ \\
H. meeusei & Yellow (maroon) & $24 \mathrm{a}$ \\
H. meraukensis & Light pink & $30 \mathrm{~b}$ \\
H. moscheutos & White (maroon) & $124 \mathrm{k}$ \\
H. nigricaulis & Yellow (maroon) & $24 \mathrm{a}$ \\
H. physaloides & Yellow (maroon) & $68 \mathrm{f}$ \\
H. radiatus & Maroon & $74 \mathrm{~g}$ \\
H. sabdariffa & Cream (maroon) & $47 \mathrm{~d}$ \\
H. schinzii & Yellow (red-brown) & $35 \mathrm{c}$ \\
H. surattensis & Yellow (maroon) & $37 \mathrm{c}$ \\
H. trionum 'Sunnyday' & Cream (maroon) & $43 \mathrm{~d}$ \\
\end{tabular}

${ }^{\mathrm{z}}$ Numerals represent species mean.

yMean separation by Tukey's Studentized range test, $P<0.05$.

leaf unfolding (Tables 2 and 3). For instance, growth of $H$. laevis and $H$. moscheutos ceased under the SD lighting treatment after plants unfolded about 10 leaves. Hibiscus aculeatus plants (roots and shoots) died when grown under the SD lighting treatment, regardless of temperature. Plants of all three species flowered only under the NI or CT lighting treatments. Leaf number below the first flower was similar under NI or CT for all three species.

Hibiscus trionum 'Sunnyday' had six leaves below the first flower bud, regardless of the light/temperature treatment. However, the first flower buds aborted under SD and NI treatments at 20 and $25^{\circ} \mathrm{C}$, resulting in variation in leaf number below the first flower to develop completely to anthesis and days to anthesis
(Tables 2 and 3). For instance, plants grown under the CT lighting treatment had about six leaves below the first flower to open regardless of temperature. Similarly, plants grown at $15^{\circ} \mathrm{C}$ had about seven leaves below the first flower to open regardless of the lighting treatment. However, plants grown at 20 or $25^{\circ} \mathrm{C}$ had a lower leaf number below the first flower to open when grown under the $\mathrm{CT}$ lighting treatment than when grown under the NI or SD lighting treatments. Similarly, plants grown at 20 or $25^{\circ} \mathrm{C}$ had a lower leaf number below first flower to open when grown under the NI than the SD lighting treatment.

Temperature interacted with lighting treatment to affect leaf number below the first flower and days to anthesis on $H$. cannabinus, $H$. mastersianus, $H$. nigricaulis, and $H$. physaloides (Tables 2 and 3). For instance, leaf number of $H$. mastersianus below the first flower when grown under the SD lighting treatment was 7 and 12 leaves on plants grown at 20 and $25{ }^{\circ} \mathrm{C}$, respectively. Similarly, $H$. mastersianus plants grown under the NI lighting treatment unfolded 10 and 23 leaves before forming a flower at 20 and $25^{\circ} \mathrm{C}$, respectively.

FLOWER COLOR AND DIAMETER. Flowers of 12 of the 21 species that flowered were yellow with maroon, or sometimes brown, centers (Table 4). Other flower colors observed were pink, white, and maroon. Flower diameter at $20^{\circ} \mathrm{C}$ under the lighting treatment that resulted in earliest flowering for each species ranged from $24 \mathrm{~mm}$ for $H$. meeusei and $H$. nigricaulis, to $124 \mathrm{~mm}$ for $H$. moscheutos (Table 4).

Temperature interacted with lighting treatment to affect flower diameter of two species, $H$. radiatus and $H$. nigricaulis. $H$. radiatus flower diameter under the SD lighting treatment at 15 , 20 , and $25^{\circ} \mathrm{C}$ was 31,72 , and $100 \mathrm{~mm}$, respectively. Increasing temperature from 20 to $25{ }^{\circ} \mathrm{C}$ increased $\mathrm{H}$. nigricaulis flower diameter from 24 to $54 \mathrm{~mm}$ under SD.

Lateral ShOOT NUMber and Plant height. Hibiscus sabdariffa, $H$. surattensis, and $H$. trionum were the only species with lateral shoots. Increasing temperature increased the number of lateral shoots for all three species when grown under the CT lighting treatment (Table 5). For example, $H$. surattensis had 0 , 4 , and 7 lateral shoots under $\mathrm{CT}$ at 15,20 , and $25^{\circ} \mathrm{C}$, respectively. In contrast, $H$. surattensis plants had a similar number of lateral shoots under all three lighting treatments at $20^{\circ} \mathrm{C}$. However, plants grown at $25^{\circ} \mathrm{C}$ had 2, 6, and 7 lateral shoots when grown under the SD, NI, and CT lighting treatments, respectively.

Species, lighting treatment, and temperature interacted to affect

Table 5. Effect of lighting and temperature treatment on lateral shoot number of three Hibiscus sp. Lighting treatments: $9 \mathrm{~h}$ ambient light (St. Paul, Minn., from November to April) (SD); ambient light plus $2 \mu \mathrm{mol} \cdot \mathrm{m}^{-2} \cdot \mathrm{s}^{-1}$ from 2200 to $0200 \mathrm{HR}$ (NI), or ambient light plus $100 \mu \mathrm{mol} \cdot \mathrm{m}^{-2} \cdot \mathrm{s}^{-1}$ continuous light (CT).

\begin{tabular}{|c|c|c|c|c|}
\hline \multirow[b]{2}{*}{ Species } & \multirow[b]{2}{*}{ Temp $\left({ }^{\circ} \mathrm{C}\right)$} & \multicolumn{3}{|c|}{ Lateral shoot no. } \\
\hline & & SD & NI & $\mathrm{CT}$ \\
\hline \multirow[t]{3}{*}{ H. sabdariffa } & 15 & Dead & Dead & Dead \\
\hline & 20 & $1^{\mathrm{z}} \mathrm{a}^{\mathrm{y}} \mathrm{A}^{\mathrm{x}}$ & $5 \mathrm{bA}$ & $4 \mathrm{bA}$ \\
\hline & 25 & $0 \mathrm{aA}$ & $7 \mathrm{bB}$ & $8 \mathrm{bB}$ \\
\hline \multirow{3}{*}{ H. surattensis } & 15 & Dead & Dead & $0 \mathrm{~A}$ \\
\hline & 20 & $4 \mathrm{aB}$ & $4 \mathrm{aA}$ & $4 \mathrm{aB}$ \\
\hline & 25 & $2 \mathrm{aA}$ & $6.0 \mathrm{bB}$ & $7 \mathrm{bC}$ \\
\hline \multirow[t]{3}{*}{ H. trionum 'Sunnyday' } & 15 & $5 \mathrm{bA}$ & $3 \mathrm{bA}$ & $2 \mathrm{aA}$ \\
\hline & 20 & $5 \mathrm{bA}$ & $2 \mathrm{aA}$ & $3 \mathrm{aB}$ \\
\hline & 25 & $7 \mathrm{bA}$ & $4 \mathrm{aA}$ & $5 \mathrm{aB}$ \\
\hline
\end{tabular}

\footnotetext{
${ }^{\mathrm{z}}$ Numerals represent treatment mean.
}

'Lower case letters indicate mean separation across lighting treatment, within a species and temperature, by Tukey's studentized range test, $P<0.05$. 'Upper case letters indicate mean separation across temperature, within a species and lighting treatment, by Tukey's studentized range test, $P<$ 0.05 . 
plant height at anthesis (Table 6). For instance, $H$. asper height at anthesis was 247,332 , and $789 \mathrm{~mm}$ under SD when grown at 15,20 , and $25^{\circ} \mathrm{C}$, respectively. Height of $H$. radiatus at anthesis at $15^{\circ} \mathrm{C}$ was 217,342, and 506 mm under SD, NI, and CT, respectively. Plant height at anthesis across all treatments ranged from $150 \mathrm{~mm}$ for $H$. costatus to $1500 \mathrm{~mm}$ for $H$. acetosella (Table 6).

\section{Discussion}

Fifteen species did not flower in any treatment after 20 weeks (Table 1). This is not surprising considering many of these species are shrubs or trees in their indigenous habitats and may have a juvenile period longer than the length of the experiment (Salisbury and Ross, 1992).

Variation in the photoperiodic requirement for flower induc- tion among Hibiscus sp. studied herein is consistent with results reported for other Hibiscus sp. (Menzel et al. ,1986; Thomas and Vince-Prue, 1997; Wilkins and Kotecki, 1982) and other genera, including Allium L. and Coleus Lour. (Thomas and Vince-Prue, 1997). For instance, interrupting a $15 \mathrm{~h}$ night $\geq 1 \mathrm{~h}$ with a low irradiance ( 1 to $3 \mu \mathrm{mol} \cdot \mathrm{m}^{-2} \cdot \mathrm{s}^{-1}$ from incandescent lamps) induced $90 \%$ of a $H$. moscheutos 'Disco Belle Mixed' population to flower (Runkle et al., 1998). Results herein support the classification of $H$. moscheutos as a long-day plant. However, subsequent work we conducted indicated $H$. moscheutos 'Disco Belle Pink' is an obligate long-day plant only when daily light integral is $\leq 13 \mathrm{~mol} \cdot \mathrm{m}^{-2} \cdot \mathrm{d}^{-1}$ (Warner, 1999). Hibiscus moscheutos 'Disco Belle Pink' is a facultative long-day plant when daily light integral is $\geq 13 \mathrm{~mol} \cdot \mathrm{m}^{-2} \cdot \mathrm{d}^{-1}$ under a $9 \mathrm{~h}$ photoperiod.

Our classification of $H$. cannabinus, $H$. mastersianus, $H$.

Table 6. Effect of temperature and lighting treatment on plant height $(\mathrm{mm})$ at anthesis of 17 Hibiscus $\mathrm{sp}$. Plants were grown under $9 \mathrm{~h}$ ambient light (St. Paul, Minn., from November to May) (SD), ambient light plus $2 \mu \mathrm{mol} \cdot \mathrm{m}^{-2} \cdot \mathrm{s}^{-1}$ from 2200 to $0200 \mathrm{HR}$ (NI), or ambient light plus $100 \mu \mathrm{mol} \cdot \mathrm{m}^{-2} \cdot \mathrm{s}^{-1}$ continuous light (CT).

\begin{tabular}{|c|c|c|c|c|}
\hline \multirow[b]{2}{*}{ Species } & \multirow{2}{*}{$\begin{array}{c}\text { Air } \\
\text { temp }\left({ }^{\circ} \mathrm{C}\right)\end{array}$} & \multicolumn{3}{|c|}{ Plant ht (mm) } \\
\hline & & SD & $\mathrm{NI}$ & $\mathrm{CT}$ \\
\hline \multirow[t]{2}{*}{ H. acetosella } & 20 & $488^{z} a^{y} A^{x}$ & $946 \mathrm{bA}$ & $568 \mathrm{a}$ \\
\hline & 25 & $494 \mathrm{aA}$ & $1500 \mathrm{bB}$ & $\mathrm{dnf}^{\mathrm{w}}$ \\
\hline \multirow[t]{2}{*}{ H. aculeatus } & 20 & $\operatorname{dnf}$ & $578 \mathrm{aA}$ & $668 \mathrm{aA}$ \\
\hline & 25 & $\mathrm{dnf}$ & $895 \mathrm{aB}$ & $790 \mathrm{aB}$ \\
\hline \multirow[t]{3}{*}{ H. asper } & 15 & $247 \mathrm{~A}$ & $\operatorname{dnf}$ & $\operatorname{dnf}$ \\
\hline & 20 & $332 \mathrm{~A}$ & dnf & dnf \\
\hline & 25 & $789 \mathrm{~B}$ & dnf & dnf \\
\hline H. calyphyllus & 20 & $256 \mathrm{a}$ & $256 a$ & $244 \mathrm{a}$ \\
\hline H. cisplatinus & 20 & $485 \mathrm{a}$ & $507 \mathrm{a}$ & $477 \mathrm{a}$ \\
\hline H. costatus & 20 & $150 \mathrm{a}$ & $273 \mathrm{~b}$ & $--^{v}$ \\
\hline H. engleri & 20 & $289 \mathrm{a}$ & $278 \mathrm{a}$ & --- \\
\hline \multirow[t]{2}{*}{ H. laevis } & 20 & $\operatorname{dnf}$ & $527 \mathrm{aA}$ & $551 \mathrm{aA}$ \\
\hline & 25 & $\operatorname{dnf}$ & $672 \mathrm{bB}$ & $611 \mathrm{aB}$ \\
\hline H. lunariifolius & 20 & $263 \mathrm{a}$ & $310 \mathrm{a}$ & --- \\
\hline \multirow[t]{2}{*}{ H. mastersianus } & 20 & $909 \mathrm{aA}$ & $972 \mathrm{bA}$ & $915 \mathrm{a}$ \\
\hline & 25 & $1079 \mathrm{aB}$ & $1338 \mathrm{bB}$ & $\operatorname{dnf}$ \\
\hline \multirow[t]{2}{*}{ H. meeusei } & 20 & $172 \mathrm{~A}$ & $\operatorname{dnf}$ & --- \\
\hline & 25 & $230 \mathrm{~A}$ & $\operatorname{dnf}$ & --- \\
\hline H. meraukensis & 20 & $162 \mathrm{a}$ & $272 b$ & $285 \mathrm{~b}$ \\
\hline \multirow[t]{3}{*}{ H. moscheutos } & 15 & $\operatorname{dnf}$ & $522 \mathrm{~A}$ & --- \\
\hline & 20 & $\mathrm{dnf}$ & $611 \mathrm{bB}$ & $545 \mathrm{aA}$ \\
\hline & 25 & $\mathrm{dnf}$ & $637 \mathrm{bB}$ & $563 \mathrm{aA}$ \\
\hline \multirow[t]{2}{*}{ H. nigricaulis } & 20 & $222 \mathrm{aA}$ & $409 \mathrm{cA}$ & $348 \mathrm{bA}$ \\
\hline & 25 & $523 \mathrm{aB}$ & $633 \mathrm{aB}$ & $1072 \mathrm{bB}$ \\
\hline \multirow[t]{2}{*}{ H. physaloides } & 20 & $729 \mathrm{~A}$ & $\operatorname{dnf}$ & $\operatorname{dnf}$ \\
\hline & 25 & $814 \mathrm{~B}$ & $\operatorname{dnf}$ & $\operatorname{dnf}$ \\
\hline \multirow[t]{3}{*}{ H. radiatus } & 15 & $217 \mathrm{aA}$ & $342 \mathrm{~b}$ & $506 \mathrm{c}$ \\
\hline & 20 & $528 \mathrm{C}$ & $\operatorname{dnf}$ & $\operatorname{dnf}$ \\
\hline & 25 & $369 \mathrm{~B}$ & $\operatorname{dnf}$ & $\mathrm{dnf}$ \\
\hline \multirow[t]{2}{*}{ H. sabdariffa } & 20 & $176 \mathrm{~A}$ & $\mathrm{dnf}$ & $\mathrm{dnf}$ \\
\hline & 25 & $198 \mathrm{~A}$ & $\operatorname{dnf}$ & $\operatorname{dnf}$ \\
\hline H. schinzii & 20 & $181 \mathrm{a}$ & $183 \mathrm{a}$ & --- \\
\hline \multirow[t]{2}{*}{ H. surattensis } & 20 & $307 \mathrm{aA}$ & $\operatorname{dnf}$ & $280 \mathrm{aA}$ \\
\hline & 25 & $570 \mathrm{bB}$ & $\operatorname{dnf}$ & $479 \mathrm{bB}$ \\
\hline
\end{tabular}

\footnotetext{
${ }^{\mathrm{z} N u m e r a l s ~ r e p r e s e n t ~ t r e a t m e n t ~ m e a n . ~}$
}

'Lower case letters indicate mean separation within a species and temperature, across lighting treatment, by Tukey's studentized range test, $P<0.05$. ${ }^{x}$ Upper case letters indicate mean separation, within a species and lighting treatment, across temperature, by Tukey's studentized range test, $P<0.05$. wPlants did not flower in this treatment.

vPlants were not grown in this treatment. 
sabdariffa, and $H$. surattensis as short-day plants is consistent with previous results (Crane, 1949; Crane and Acuna, 1945; Menzel et al., 1986). Hibiscus surattensis flowered under both SD and CT, but flowering was completely inhibited by NI. Inhibition of flowering by NI, but not SD or CT, has been reported for other short-day plants, including $H$. sabdariffa (El-Afry et al., 1980; Mansour, 1975). However, for results presented herein, $H$. sabdariffa did not flower when grown under CT. Similarly, Cockshull (1979) reported that three cultivars of the short-day plant, Dendranthema $\times$ grandiflorum Kitam. (syn. Chrysanthemum $\times$ morifolium Ramat.) (chrysanthemum), initiated flower buds when grown under continuous light. However, flower buds of only one of the three cultivars, 'Golden Stardust', developed to anthesis, and only when the temperature was 10 or $16^{\circ} \mathrm{C}$.

The photoperiodic response for flowering of a given species is closely linked to the indigenous habitat of that species. Plants from latitudes 30 to $55^{\circ} \mathrm{N}$ are often long-day plants that may or may not require vernalization (Roberts and Summerfield, 1987). This ensures that plants will flower and produce seed during the warm summer months before going dormant for the winter. In contrast, plants from latitudes of 0 to $30^{\circ} \mathrm{N}$ are often short-day plants or day-neutral (Roberts and Summerfield, 1987). Our results are consistent with observations of Roberts and Summerfield (1987) where Hibiscus sp. native to the United States (i.e., $H$. militaris and $H$. moscheutos) were obligate longday plants. In contrast, species native closer to the equator (i.e., H. calyphyllus, $H$. cannabinus, and $H$. radiatus) were day-neutral and short-day plants (Tables 1 and 2).

High temperature treatment $\left(25^{\circ} \mathrm{C}\right)$ delayed flowering of some Hibiscus sp. under inductive lighting treatments (Tables 2 and 3 ). For example, increasing temperature from 20 to $25^{\circ} \mathrm{C}$ increased leaf number below the first flower on $H$. mastersianus and $H$. nigricaulis, i.e., developmental delay. In contrast, increasing temperature from 20 to $25^{\circ} \mathrm{C}$ increased leaf number below the first flower and days to anthesis on $H$. asper and $H$. physaloides, i.e., developmental delay and delay in time. In all cases, Hibiscus sp. that exhibited high temperature delay in flowering eventually flowered. Interestingly, species that exhibited high temperature delay were among facultative or obligate short-day plant groups only.

High temperature inhibition or delay of flowering has been observed in other species. Short-day induced flower initiation in Euphorbia pulcherrima Willd. ex Klotzsch. (poinsettia) is delayed when night temperature exceeds $22^{\circ} \mathrm{C}$ (Dole and Wilkins, 1999). Similarly, short-day induced flowering in Dendranthema $\times$ grandiflorum and Schlumbergera truncata (Haw.) Moran. (Thanksgiving cactus) is delayed when day or night temperature exceeds $22^{\circ} \mathrm{C}$ (Karlsson et al., 1989; Erwin et al., 1990). Pharbitis nil Chois. flowering is delayed (Reese and Erwin, 1997) and Pelargonium $\times$ hortorum L.H. Bail. (geranium) flower number per inflorescence decreases as average daily temperature increases (Erwin and Heins, 1993).

Based on subjective characteristics mentioned previously, we determined that $H$. cisplatinus and $H$. radiatus have potential as new commercial ornamental crops. Hibiscus acetosella and $H$. trionum are currently commercially available, but are not widely grown.

\section{Literature Cited}

Bates, D.M. 1965. Notes on the cultivated Malvaceae: Hibiscus. Baileya 13:56-129.

Cockshull, K.E. 1979. Effects of irradiance and temperature on flowering of Chrysanthemum morifolium Ramat. in continuous light. Ann. Bot. 44:451-460.

Crane, J.C. 1949. Roselle-A potentially important fibre crop. Econ. Bot. 3:89-103.

Crane, J.C. and J.B. Acuna. 1945. Effect of plant spacing and time of planting on seed yield of kenaf, Hibiscus cannabinus L. J. Amer. Soc. Agron. 38:969-977.

Dole, J.M. and H.F. Wilkins. 1999. Floriculture: Principles and species. Prentice-Hall, Inc., Upper Saddle River, N.J.

El-Afry, M.M.F., E.R. Khafaga, and D. Prinz. 1980. Studies on photoperiodic reaction of roselle (Hibiscus sabdariffa L. var. sabdariffa). Angew. Bot. 54:279-286.

Erwin, J.E. and R.D. Heins. 1993. Light and temperature, p. 55-63. In: J.W. White (ed.). Geraniums IV. Ball Publishing, Geneva, Ill.

Erwin, J.E., R.D. Heins, R. Berghage, and B. Kovanda. 1990. Temperature effects Sclumbergera truncata 'Madisto' flower initiation. Acta Hort. 272:97-101.

Karlsson, M.G., R.D. Heins, J.E. Erwin, R.D. Berghage, W.H. Carlson, and J.A. Biernbaum. 1989. Temperature and photosynthetic photon flux influence Chrysanthemum shoot development and flower initiation under short-day conditions. J. Amer. Soc. Hort. Sci. 114:158-163.

Mansour, B.M.M. 1975. Effects of temperature and daylength on growth and flowering of roselle, Hibiscus sabdariffa L. Scientia Hort. 3:129135 .

Menzel, M.Y., P.A. Fryxell, and F.D. Wilson. 1983. Relationships among new world species of Hibiscus section Furcaria (Malvaceae). Brittonia 35:204-221.

Menzel, M.Y., K.L. Richmond, C.S. Contolini, and P. Huang. 1986. New intergenomic hybrids among diploid species of Hibiscus sect. Furcaria. Amer. J. Bot. 73:304-309.

Menzel, M.Y. and F.D. Wilson. 1963. Cytotaxonomy of twelve species of Hibiscus section Furcaria. Amer. J. Bot. 50:262-271.

Nau, J. 1996. Ball perennial manual: Propagation and production. Ball Publishing. Batavia, Ill.

Reese, C.R. and J.E. Erwin. 1997. The effect of day/night temperature on Pharbitis nil Chois. flowering. HortScience 32:1046-1048.

Rickett, H.M. 1970. Wildflowers of the United States. vol. 4. The southwestern states. Part I. McGraw-Hill, New York.

Roberts, E.H. and R.J. Summerfield. 1987. Measurement and prediction of flowering in annual crops, p. 17-50. In: J.G. Atherton (ed.). Manipulation of flowering. Butterworths, London.

Runkle, E.S., R.D. Heins, A.C. Cameron, and W.H. Carlson. 1998. Flowering of herbaceous perennials under various night interruption and cyclic lighting treatments. HortScience 33:672-677.

Runkle, E.S., R.D. Heins, W. Carlson, and A. Cameron. 1996. Manipulating daylength to flower perennials. Grower Talks 60:66-70.

Salisbury,F.B. and C.W. Ross. 1992.Plant physiology. 4thed. Wadsworth Publishing Co., Belmont, Calif.

SPSS, Inc. 1997. SPSS base 7.5 for windows user's guide. SPSS, Inc., Chicago, Ill.

Thomas, B. and D. Vince-Prue. 1997. Photoperiodism in plants. 2nd ed. Academic Press, San Diego, Calif.

Warner, R.M. 1999. Temperature and light effects on flowering of Hibiscus spp. MS thesis., Univ. of Minn., Saint Paul.

Wilkins, H.F. and D. Kotecki. 1982. Hibiscus rosa-sinensis L. Minn. State Florists Bul. 31:3-7.

Wilson, F.D. 1974. Hibiscus section Furcaria (Malvaceae) in Australia. Austral. J. Bot. 22:157-182. 